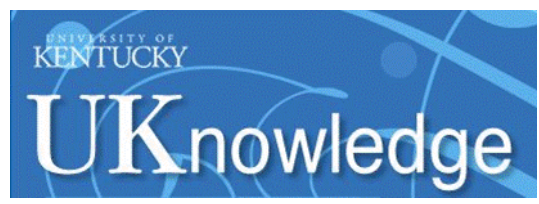

University of Kentucky

UKnowledge

$12-9-2014$

\title{
Collective Edge Modes Near the Onset of a Graphene Quantum Spin Hall State
}

\author{
Gunpathy Murthy \\ University of Kentucky, murthy@pa.uky.edu \\ Efrat Shimshoni \\ Bar-Ilan University, Israel \\ H. A. Fertig \\ Indiana University
}

Follow this and additional works at: https://uknowledge.uky.edu/physastron_facpub

Part of the Astrophysics and Astronomy Commons, and the Physics Commons

Right click to open a feedback form in a new tab to let us know how this document benefits you.

\section{Repository Citation}

Murthy, Gunpathy; Shimshoni, Efrat; and Fertig, H. A., "Collective Edge Modes Near the Onset of a Graphene Quantum Spin Hall State" (2014). Physics and Astronomy Faculty Publications. 286. https://uknowledge.uky.edu/physastron_facpub/286

This Article is brought to you for free and open access by the Physics and Astronomy at UKnowledge. It has been accepted for inclusion in Physics and Astronomy Faculty Publications by an authorized administrator of UKnowledge. For more information, please contact UKnowledge@lsv.uky.edu. 


\section{Collective Edge Modes Near the Onset of a Graphene Quantum Spin Hall State}

Digital Object Identifier (DOI)

http://dx.doi.org/10.1103/PhysRevB.90.241410

Notes/Citation Information

Published in Physical Review B: Condensed Matter and Materials Physics, v. 90, no. 24, article 241410, p. 1-5.

(C)2014 American Physical Society

The copyright holder has granted permission for posting the article here. 


\title{
Collective edge modes near the onset of a graphene quantum spin Hall state
}

\author{
Ganpathy Murthy, ${ }^{1}$ Efrat Shimshoni, ${ }^{2}$ and H. A. Fertig ${ }^{3}$ \\ ${ }^{1}$ Department of Physics and Astronomy, University of Kentucky, Lexington, Kentucky 40506-0055, USA \\ ${ }^{2}$ Department of Physics, Bar-Ilan University, Ramat-Gan 52900, Israel \\ ${ }^{3}$ Department of Physics, Indiana University, Bloomington, Indiana 47405, USA \\ (Received 4 September 2014; revised manuscript received 22 October 2014; published 9 December 2014)
}

\begin{abstract}
Graphene subject to a strong, tilted magnetic field exhibits an insulator-metal transition tunable by tilt angle, attributed to the transition from a canted antiferromagnetic (CAF) to a ferromagnetic (FM) bulk state at filling factor $v=0$. We develop a theoretical description for the spin and valley edge textures in the two phases, and the implied evolution in the nature of edge modes through the transition. In particular, we show that the CAF has gapless neutral modes in the bulk, but supports gapped charged edge modes. At the transition to the FM state the charged edge modes become gapless and are smoothly connected to the helical edge modes of the FM state. Possible experimental consequences are discussed.
\end{abstract}

DOI: 10.1103/PhysRevB.90.241410

PACS number(s): 73.21.-b, 72.80.Vp, 73.22.Gk, 73.43.Lp

Introduction. Graphene subject to a perpendicular magnetic field exhibits a quantum Hall $(\mathrm{QH})$ state at $v=0$, made possible by electron-electron interactions [1-14]. The emergence of a $\sigma_{x y}=0$ plateau indicates the presence of a bulk gap in the half-filled zero Landau level associated with the formation of a broken-symmetry many-body state. The variety of different ways to spontaneously break the $S U(4)$ symmetry in spin and valley space suggests a plethora of possible ground states [15-21], of which the favored one is dictated by the combined effect of interactions and external fields. Most notably, a phase transition has been proposed [18,22] from a canted antiferromagnetic (CAF) to a spin-polarized ferromagnetic (FM) state tuned by increasing the Zeeman energy $E_{z}$ to appreciable values. Both phases are, in principle, accessible in strong, tilted magnetic fields, and differ in fundamental ways: The CAF is an insulator, characterized by gapped charged excitations on the edge [23]. By contrast the FM state supports gapless, helical, charged excitations at its edge [24-27]. In this work we address how the edge excitations reflect the differing characters of these states, and how they continuously evolve into one another as the system passes through the quantum phase transition between them.

A recent experiment by Young et al. [28] shows the CAF-FM transition in transport measurements, performed in magnetic fields tilted with respect to the graphene plane. For a fixed perpendicular component of the total magnetic field $B_{T}$, increasing $E_{z} \propto B_{T}$ beyond a critical value $E_{z}^{c}$ drives the $v=0$ state from an insulator (with two-terminal conductance $G=0)$ to an almost perfect conductor $\left(G \lesssim 2 e^{2} / h\right)$. This can be attributed to the change in the corresponding edge states. In analogy with the quantum spin Hall (QSH) state in two-dimensional topological insulators [29,30], the gapless edge states of the FM state are immune to backscattering by spin-conserving impurities due to their helical nature: right and left movers have opposite spin flavors.

In a noninteracting model [24], the edge modes of the QSH state are one-dimensional (1D) single-electron channels, centered at crossing points of dispersing energy levels with opposite spin index. However, interactions introduce a finite spin stiffness and lead to the formation of a coherent domain wall (DW), and a gap to particle-hole excitations. The low-energy charged excitations are gapless collective modes associated with fluctuations of the ground-state spin configuration, in the form of a $2 \pi$ rotation in the $\left(s_{x}, s_{y}\right)$ plane [25]. This spin twist is imposed upon the position-dependent $s_{z}$ associated with the DW, thus creating a spin texture, with an associated charge that is inherent to $\mathrm{QH}$ ferromagnets [31,32]. Gapless 1D modes of the DW (which can be modeled as a helical Luttinger liquid [26]) carry charge and contribute to electric conduction. As spin waves in the FM bulk are gapped, their interaction with the gapless edge modes has a minor effect on the 1D dynamics [33] and the resulting transport behavior.

In contrast, the CAF phase is characterized by a gap to charged excitations on the edge [23,34], and a broken $U$ (1) symmetry in the bulk (associated with $X Y$-like order parameter) implying a neutral, gapless bulk Goldstone mode. As described below, a proper description of the lowest-energy charged excitations of this state involves a coupling between topological structures at the edge and in the bulk, associated with the broken $U(1)$ symmetry. This is particularly crucial in proximity to the CAF-FM transition, where the bulk stiffness softens and ultimately controls the energetics of charged excitations.

In this work, we theoretically describe the evolution of collective edge excitations as $E_{z}$ is tuned across the CAFFM transition. Our approach significantly generalizes the mean-field ansatz of Ref. [18] in a way that allows the bulk and edge of the system to be treated on an equal footing. Based upon numerical Hartree-Fock calculations, we derive a simple description for a spin-valley domain wall configuration at the edge for arbitrary $E_{z}$, parametrized by two canting angles $\psi_{a}, \psi_{b}$ (see Fig. 1) which characterize how the two occupied $n=0$ Landau levels of the $v=0$ state evolve as one approaches an edge. Low-energy charged excitations can be constructed by imposing a slowly varying spin rotation on this state. In the CAF, these involve binding a vortex (meron) of the bulk state to a spin twist at the edge, so that the bulk spin stiffness controls the excitation energy. As the CAF-FM transition is approached, the bulk stiffness vanishes and the bound vortex "evaporates," yielding a gapless edge excitation [25]. Our model predicts the behavior of the activation gap in edge transport as a function of $E_{z}$ and offers a qualitative 


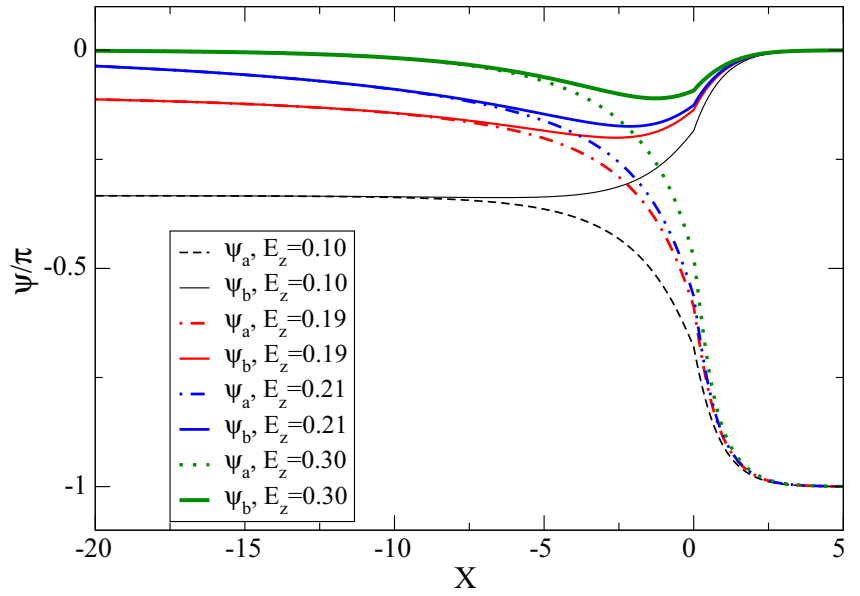

FIG. 1. (Color online) Variation of the canting angles $\psi_{a}, \psi_{b}$ [see Eq. (3)] with guiding center $X$ (in units of $\ell$ ), in the presence of an edge potential near $X=0$. The critical Zeeman energy for CAFFM transition is $E_{z}^{c}=0.2$. The results are obtained from numerical Hartree-Fock calculations with the maximum edge potential being $U_{e}=5$ and the width of the edge being $w=3 \ell$.

picture of how this transport should evolve with filling factor. Further experimental consequences of our model are discussed below.

Hartree-Fock analysis and edge structure. We consider a monolayer of graphene uniform in the $y$ direction, and subject to an edge potential $U(x)$ which grows linearly over a length scale $w$ from zero in the bulk $(x \leqslant 0)$ to a constant $U_{e}$ for $x>w$. The system is subject to a tilted magnetic field of magnitude $B_{T}$ (dictating the Zeeman energy $E_{z}$ ) and perpendicular component $B_{\perp}$. The single-electron states are labeled by a guiding-center coordinate $X$. For a given $X$, there are four orthogonal states in the full $n=0$ Landau level. Their wave function can be written in a basis of four-spinors $|X s \tau\rangle$ where $s=\uparrow, \downarrow$ denotes the real spin index $s_{z}$, and $\tau= \pm$ is an isospin, corresponding to symmetric and antisymmetric combinations of valley states. The latter are eigenvalues of $\hat{\tau}_{x}$, and for convenience our single-particle Hamiltonian implements a simplified edge potential proportional to this operator [23]. At zero doping, two of the four Landau levels are filled.

Our model Hamiltonian is projected into the manifold of $n=0$ states labeled by $X$ and has the form

$$
H=\sum_{X} c^{\dagger}(X)\left[-E_{z} \sigma_{z} \tau_{0}+U(X) \sigma_{0} \tau_{x}\right] c(X)+H_{\mathrm{int}},
$$

where $c^{\dagger}(X), c(X)$ are four-spinor operators, $\sigma_{\alpha}\left(\tau_{\alpha}\right)$ are the spin (isospin) Pauli matrices, $\sigma_{0}$ and $\tau_{0}$ are unit matrices, and $H_{\text {int }}$ is the interaction term. In analogy with Refs. [18,23], we assume that $H_{\text {int }}$ contains short-range interactions which may break the $S U(4)$ symmetry, but we also retain an $S U(4)$ symmetric contribution [35] (which provides the crucial spin stiffness required to stabilize inhomogeneous solutions), so that $H_{\text {int }}$ takes the form

$$
H_{\text {int }}=\frac{\pi \ell^{2}}{L^{2}} \sum_{\alpha=0, x, y, z} \sum_{X_{1}, X_{2}, q} e^{-q^{2} \ell^{2} / 2+i q\left(X_{1}-X_{2}\right)} g_{\alpha}: c^{\dagger}\left(X_{1}+\frac{q \ell^{2}}{2}\right) \tau_{\alpha} c\left(X_{1}-\frac{q \ell^{2}}{2}\right) c^{\dagger}\left(X_{2}-\frac{q \ell^{2}}{2}\right) \tau_{\alpha} c\left(X_{2}+\frac{q \ell^{2}}{2}\right):
$$

in which we assume $g_{x}=g_{y} \equiv g_{x y}, \ell=\sqrt{\hbar c / e B_{\perp}}$ is the magnetic length, $L$ is the system size, and : : denotes normal ordering. We presume in what follows that the short-range interactions satisfy $g_{z}>-g_{x y}>0$, which is required to stabilize a CAF state for small $E_{z}$ [18].

Within a set of single Slater determinant (Hartree-Fock) states, with two states occupied within the four-dimensional space for each $X$, we find numerically that for arbitrary $E_{z}$ and edge potential $U(X)$ the energy is minimized by a remarkably simple ansatz for the two filled states, denoted as $\left|a_{X}\right\rangle$ and $\left|b_{X}\right\rangle$ :

$$
\begin{aligned}
& \left|a_{X}\right\rangle=\cos \left[\psi_{a}(X) / 2\right]|X \uparrow+\rangle-\sin \left[\psi_{a}(X) / 2\right]|X \downarrow-\rangle, \\
& \left|b_{X}\right\rangle=-\cos \left[\psi_{b}(X) / 2\right]|X \uparrow-\rangle+\sin \left[\psi_{b}(X) / 2\right]|X \downarrow+\rangle .
\end{aligned}
$$

$\psi_{a}(X)$ and $\psi_{b}(X)$ represent canting angles of the spin, which vary continuously as a function of $X$, and are generally different as depicted in Fig. 1.

In the bulk ( $X \ll 0$ in Fig. 1 ) we recover the configuration found in Ref. [18]: $\psi_{a}=\psi_{b}=\psi$, where for $E_{z}<E_{z}^{c}=$ $2\left|g_{x y}\right|$ a CAF is established with $\psi$ obeying $\cos \psi=E_{z} / E_{z}^{c}$, while for $E_{z}>E_{z}^{c}$ (the FM phase), $\psi=0$. However, $\psi_{a, b}$ deviate from this uniform solution near the edge, smoothly approaching an isospin-polarized, spin singlet state with $\psi_{a}=$ $-\pi, \psi_{b}=0$ for large $U_{e}$. In the intermediate region, spin and isospin are entangled. It should also be noted that for nontrivial canting angles $\left(\psi_{a, b} \neq n \pi\right)$, a manifold of degenerate solutions exists with relative phase factors $e^{i \phi}$ between the $s=\uparrow$, $s=\downarrow$ spin components, implying a spontaneously broken $U(1)$ symmetry and associated gapless Goldstone modes. For the bulk CAF state these are gapless spin-wave excitations in the bulk. For the FM state, one finds gapless states at the edge, which moreover can be used to construct gapless charged excitations [25].

Note that the spatial scale of the edge structure becomes arbitrarily large as $E_{z} \rightarrow E_{z}^{c}$. This may be understood by evaluating the expectation value of the Hamiltonian [Eq. (1)] in the state of Eq. (3). Defining $\psi=\left(\psi_{a}+\psi_{b}\right) / 2$ and $\chi=$ $\left(\psi_{a}-\psi_{b}\right) / 2$, and assuming slowly varying $\psi$ and $\chi$ we get

$$
E_{\mathrm{HF}} \approx A(\psi, \chi)+B_{\psi}(\psi, \chi)\left(\psi^{\prime}\right)^{2}+B_{\chi}(\psi, \chi)\left(\chi^{\prime}\right)^{2} .
$$

For the FM state, $\psi=\chi=0$, so that we expect these quantities to be small in the part of the bulk nearest the edge. Expanding to quadratic order and dropping an overall constant, one finds $A \approx\left[E_{z}+2 g_{x y}\right] \psi^{2}+\left[E_{z}+g_{x y}+g_{z}\right] \chi^{2}, B_{\psi} \approx$ $\left[g_{0}+g_{z}-3 g_{x y}\right] / 4$, and $B_{\chi} \approx\left[g_{0}-2 g_{z}-g_{x y}\right] / 4$. This form of the energy functional implies that $\psi$ and $\chi$ will decay into 
the bulk with length scales

$$
\ell_{\psi}=\sqrt{\frac{g_{0}+g_{z}-3 g_{x y}}{E_{z}+2 g_{x y}}}, \quad \ell_{\chi}=\sqrt{\frac{g_{0}-2 g_{z}-g_{x y}}{E_{z}+g_{z}+g_{x y}}} .
$$

Note that $\ell_{\psi} \rightarrow \infty$ for $E_{z} \rightarrow E_{z}^{c} \equiv-2 g_{x y}$, indicating a divergent length scale at the edge as the bulk transition is approached. An analogous divergent length scale is realized on the CAF side of the transition. Thus, the CAF phase penetrates into the bulk FM phase from the edge as $E_{z}$ is lowered towards the critical value.

Charged excitations: Merons and edge solitons. In a quantum Hall ferromagnet, low-energy charged excitations may be constructed from slow gradients in the various phase angles $\left(\psi_{a}, \psi_{b}\right.$, and $\left.\phi\right)$ in which most of the system is locally in a ground-state configuration [31,32]. Vortexlike excitations of these systems have nontrivial core structures and are generically known as merons. One approach to evaluating their charge is by explicit construction of wave functions with the appropriate topology. In the present context these can be written in the form

$$
\begin{aligned}
|\Phi\rangle= & \prod_{\alpha, \alpha^{\prime}}\left[u_{a}(\alpha) c_{\downarrow,-}^{\dagger}(\alpha)+v_{a}(\alpha) c_{\uparrow,+}^{\dagger}\left(\alpha_{+}\right)\right] \\
& \times\left[u_{b}\left(\alpha^{\prime}\right) c_{\downarrow,+}^{\dagger}\left(\alpha^{\prime}\right)+v_{b}\left(\alpha^{\prime}\right) c_{\uparrow,-}^{\dagger}\left(\alpha_{+}^{\prime}\right)\right]|0\rangle,
\end{aligned}
$$

where the index $\alpha$ represents the angular momentum (integer) quantum number $m$, and $\alpha_{+}=m+1$; the coherent combination of $m$ and $m+1$ angular momenta in the single-particle states implements the vorticity of the in-plane spins [32]. (The opposite vorticity is implemented by coupling the $m$ and $m-1$ states.) The $u_{a}, v_{a}, u_{b}, v_{b}$ coefficients must tend to their ground-state values as $m \rightarrow \infty\left(-\sin \left[\psi_{a} / 2\right], \cos \left[\psi_{a} / 2\right]\right.$, $\sin \left[\psi_{b} / 2\right]$, and $-\cos \left[\psi_{a} / 2\right]$, respectively). For $m \rightarrow 0$, definite vorticity implies $u \rightarrow 0$ or $v \rightarrow 0$. The evolution of the $u$ 's and $v$ 's with increasing $m$ will be smooth in a low-energy state (and should occur over the very long length scale $\sim \ell_{\psi}$ near the FM-CAF transition), so one may graphically represent these two possibilities as depicted in Fig. 2(a).

The transfer of weight from $m$ to $m+1$ with increasing $m$ leads to a deficit or excess of charge relative to the ground state. For the two examples illustrated in Fig. 2(a) these charges are $-\sin ^{2}(\psi / 2)$ when the spin is polarized downward for $m=0$, and $\cos ^{2}(\psi / 2)$ when polarized upward. (For states of the opposite vorticity, the signs of these charges are reversed.) Noting that $\psi_{a}$ and $\psi_{b}$ can independently be polarized upward or downward in a meron core, in the CAF state one finds three possible charges, $\cos \psi_{a}-\mu$, with $\mu=-1,0,1$ (we have used the property $\psi_{a}=\psi_{b}$ in the CAF ground state).

In the bulk of the CAF state, the $X Y$-like order parameter and the finite spin stiffness mean that isolated merons have logarithmically divergent energy. Charged excitations of finite energy in the bulk CAF can be generated by combining meronantimeron pairs with charges $0, \pm 1$, and \pm 2 .

In practice, many transport experiments (e.g., Ref. [28]) are dominated by charged edge excitations, which have lower energy than those of the bulk. In the FM state, zero energy charged excitations can be generated by imposing a slow $2 \pi$ rotation of the $U(1)$ variable $\phi(\mathbf{r})$ along the edge [25]. The state representing this has the same form as Eq. (5), where

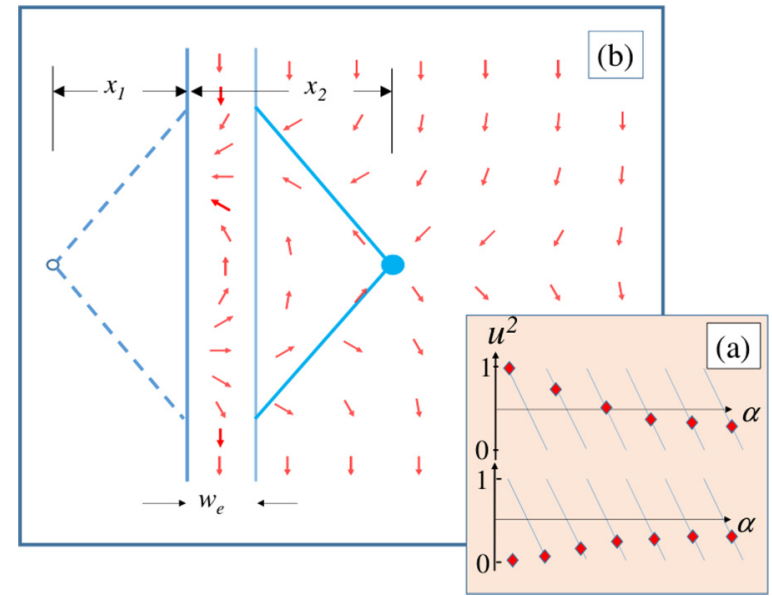

FIG. 2. (Color online) (a) Graphical representation of HartreeFock wave functions in a Landau level supporting a texture. States of different index $\alpha$ (see text) are admixed to implement a rotation in $\phi$. States near $\alpha \rightarrow 0$ must be polarized down $\left(u^{2}=1\right.$, top) or up $\left(u^{2}=0\right.$, bottom). Position of (red) diamond graphically indicates the relative weight of the admixed states as $\alpha$ increases. (b) Model of "edge soliton" consisting of image and real vortices, and in-plane $2 \pi$ spin twist at the domain wall.

the index $\alpha$ becomes the guiding center coordinate and $\alpha_{+}=$ $X+2 \pi \ell^{2} / L$. Since $\psi_{a, b} \rightarrow 0$ in the bulk for the FM state, the phase twist only changes the single-particle states near the edge, leading to a zero energy state in the thermodynamic limit.

A key question for this system is: What becomes of this gapless charged mode as one enters the CAF phase? The FM edge mode described above is a high-energy state in this case because the phase twist incurs the stiffness penalty throughout the bulk of the system: The finite amplitude of the $X Y$-like order parameter (i.e., the in-plane spin component) dictates a stiffness coefficient $\rho_{b} \propto \sin ^{2} \psi_{a} \propto\left(E_{z}^{c}-E_{z}\right)$. This divergent bulk contribution to the energy can be eliminated if the edge twist is coupled to a bulk vortex, along with an image of opposite vorticity outside the system, as illustrated in Fig. 2(b). To estimate the size scale and energy of this configuration we adopt a simple $U(1)$ energy functional of the form $E=$ $\frac{1}{2} \int d^{2} r \rho_{s}(x)|\vec{\nabla} \phi(\mathbf{r})|^{2}$, with a piecewise constant spin stiffness: $\rho_{s}(x)=0$ for $x<0, \rho_{s}(x)=\rho_{e}$ for $0<x<w_{e}$ representing the phase stiffness of the edge structure, of width $w_{e}$, and $\rho_{s}\left(x>w_{e}\right)=\rho_{b}$ represents the bulk stiffness. Taking $\phi(\mathbf{r})$ to be the sum of opposed $2 \pi$ rotations centered at distances $x_{1}<0$ and $x_{2}>w_{e}$ from the edge as illustrated in Fig. 2(b), we minimize the energy with respect to $x_{1}, x_{2}$. In the vicinity of the CAF-FM transition this yields

$$
E_{\mathrm{sol}}=\pi \rho_{b}\left[\log \left(\xi_{\mathrm{sol}} / \eta\right)+0.738\right],
$$

where $\xi_{\text {sol }}=\left(\rho_{e}-\rho_{b}\right) w_{e} / \rho_{b}$ and $\eta$ is a short distance cutoff indicating the core size of the vortex.

Several comments are in order. (i) The size scale $\xi_{\text {sol }}$ of this "edge soliton" is controlled by the bulk stiffness $\rho_{b}$. Thus the energy of the excitation vanishes as $\left(E_{z}^{c}-E_{z}\right) \log \left(E_{z}^{c}-E_{z}\right)$ as the CAF-FM transition is approached. (ii) The charge of the edge soliton is the sum of charges in the domain wall twist and the bulk meron, which is \pm 1 or 0 . (iii) In the limit $E_{z} \rightarrow E_{z}^{c}$ 
from below, the charged solitons continuously evolve into the gapless edge excitations of the FM state, with the meron portion of the excitation "evaporating" as the ground state spins become polarized along the total field direction. Thus the gapless, charged edge mode of the FM state is continuously connected to a gapped, charged edge mode of the CAF state.

Discussion. In principle, the charged soliton of the CAF state should control the activation energy for the quantized Hall effect observed in transport experiments on the CAF such as those of Ref. [28]. The general form of this energy, $E_{\text {sol }} \sim \rho_{b} \log \rho_{b}$, indicates that one may learn about the bulk phase stiffness of the CAF state via edge-dominated transport. This stiffness may be renormalized from the mean-field behavior used in the analysis above by both quantum and thermal fluctuations. An interesting possibility due to the latter of these is that the bulk should undergo a Kosterlitz-Thouless transition at some finite temperature $T_{K T}$, above which $\rho_{b}$ should jump to zero. Thus we expect that a sufficiently clean system will display cusplike behavior in its diagonal resistance as $T$ passes through the transition point. If observed, this would yield direct evidence of the broken $U(1)$ symmetry inherent to the CAF state.

Further properties of these solitons could be uncovered by studying how edge transport evolves as a function of doping, which forces them electrostatically into the ground state. At low concentrations these are presumably pinned by disorder, but at sufficiently high density, they could undergo a depinning transition, leading to dissipative transport. Interestingly, possible signatures of such a metal-insulator transition as a function of doping are evident in data presented in Ref. [28].

The FM phase of this system is a spin Hall insulator which supports a conductance of $2 e^{2} / h$ in the $T \rightarrow 0$ limit [24-26] due to the helical nature of the edge states. While measurements [28] do show a transition from a dissipationless to a dissipative state as $E_{z}$ is increased, the (extrapolated) $T=0$ conductance seems to fall short of the ideal value. While this could result from the mismatch between quasiparticle states of a metallic lead and the highly delocalized gapless charged states of the FM edge, it is interesting to speculate that intrinsic dissipation may arise from their interaction with other low-energy modes at the edge or in the bulk. The relevant mechanism explaining the shortfall in conductance can be distinguished by four terminal measurements of the diagonal conductance.

In summary, we have developed a model of the $v=0$ graphene edge, demonstrating that it supports unusual gapped charge solitons in the CAF state which continuously evolve into gapless excitations as the FM state is entered. These excitations provide information about the phase stiffness of the CAF state, and should control the low-energy behavior of the system.

Many open questions remain, such as the full structure of the edge excitations, both neutral and charged, and the effective theory of the transition. The authors plan to investigate these issues in future work.

Acknowledgments. We are grateful to A. Young, P. JarillHerrero, R. Shankar, and E. Berg for useful discussions. We would like to thank the Aspen Center for Physics (NSF Grant No. 1066293) for its hospitality. This work was partly supported by the US-Israel Binational Science Foundation via Grant No. 2012120 (E.S., G.M., H.A.F). E.S. acknowledges support by the Israel Science Foundation via Grant No. 231/14 and also travel support by the Simons Foundation. G.M. acknowledges support by the National Science Foundation via Grants No. NSF-PHY 0970069 and No. NSF-DMR 1306897. H.A.F. acknowledges support by the National Science Foundation via Grant No. NSF-DMR 1005035.
[1] Y. Zhang, Z. Jiang, J. P. Small, M. S. Purewal, Y.-W. Tan, M. Fazlollahi, J. D. Chudow, J. A. Jaszczak, H. L. Stormer, and P. Kim, Phys. Rev. Lett. 96, 136806 (2006).

[2] J. Alicea and M. P. A. Fisher, Phys. Rev. B 74, 075422 (2006).

[3] M. O. Goerbig, R. Moessner, and B. Doucot, Phys. Rev. B 74, 161407 (2006).

[4] V. P. Gusynin, V. A. Miransky, S. G. Sharapov, and I. A. Shovkovy, Phys. Rev. B 74, 195429 (2006).

[5] K. Nomura and A. H. MacDonald, Phys. Rev. Lett. 96, 256602 (2006).

[6] Z. Jiang, Y. Zhang, H. L. Stormer, and P. Kim, Phys. Rev. Lett. 99, 106802 (2007).

[7] I. F. Herbut, Phys. Rev. B 75, 165411 (2007).

[8] J. N. Fuchs and P. Lederer, Phys. Rev. Lett. 98, 016803 (2007).

[9] D. A. Abanin, K. S. Novoselov, U. Zeitler, P. A. Lee, A. K. Geim, and L. S. Levitov, Phys. Rev. Lett. 98, 196806 (2007).

[10] J. G. Checkelsky, L. Li, and N. P. Ong, Phys. Rev. Lett. 100, 206801 (2008); Phys. Rev. B 79, 115434 (2009).

[11] Xu Du, I. Skachko, F. Duerr, A. Luican, and E. Y. Andrei, Nature (London) 462, 192 (2009).

[12] M. O. Goerbig, Rev. Mod. Phys. 83, 1193 (2011).

[13] Andrea F. Young, Cory R. Dean, Lei Wang, Hechen Ren, Paul Cadden-Zimansky, Kenji Watanabe, Takashi Taniguchi, James
Hone, Kenneth L. Shepard, and Philip Kim, Nat. Phys. 8, 550 (2012).

[14] G. L. Yu, R. Jalil, Branson Belle, Alexander S. Mayorov, Peter Blake, Frederick Schedin, Sergey V. Morozov, Leonid A. Ponomarenko, F. Chiappini, S. Wiedmann, Uli Zeitler, Mikhail I. Katsnelson, A. K. Geim, Kostya S. Novoselov, and Daniel C. Elias, Proc. Natl. Acad. Sci. USA 110, 3282 (2013).

[15] I. F. Herbut, Phys. Rev. B 76, 085432 (2007).

[16] J. Jung and A. H. MacDonald, Phys. Rev. B 80, 235417 (2009).

[17] R. Nandkishore and L. S. Levitov, Phys. Scr. T 146, 014011 (2009); arXiv:1002.1966.

[18] M. Kharitonov, Phys. Rev. B 85, 155439 (2012).

[19] Bitan Roy, M. P. Kennett, and S. Das Sarma, arXiv:1406.5184.

[20] J. L. Lado and J. Fernandez-Rossier, Phys. Rev. B 90, 165429 (2014).

[21] Y. Zhao, P. Cadden-Zimansky, F. Ghahari, and P. Kim, Phys. Rev. Lett. 108, 106804 (2012).

[22] An analogous transition has been proposed for quantum Hall bilayer systems at filling factor $v=2$. See, for example, S. Das Sarma, Subir Sachdev, and Lian Zheng, Phys. Rev. Lett. 79, 917 (1997); L. Brey, ibid. 81, 4692 (1998); J. Schliemann and A. H. MacDonald, ibid. 84, 4437 (2000).

[23] M. Kharitonov, Phys. Rev. B 86, 075450 (2012). 
[24] D. A. Abanin, P. A. Lee, and L. S. Levitov, Phys. Rev. Lett. 96, 176803 (2006).

[25] H. A. Fertig and L. Brey, Phys. Rev. Lett. 97, 116805 (2006).

[26] E. Shimshoni, H. A. Fertig, and G. V. Pai, Phys. Rev. Lett. 102, 206408 (2009).

[27] M. Killi, T.-C. Wei, I. Affleck, and A. Paramekanti, Phys. Rev. Lett. 104, 216406 (2010); S. Wu, M. Killi, and A. Paramekanti, Phys. Rev. B 85, 195404 (2012).

[28] A. F. Young, J. D. Sanchez-Yamagishi, B. Hunt, S. H. Choi, K. Watanabe, T. Taniguchi, R. C. Ashoori, and P. Jarillo-Herrero, Nature (London) 505, 528 (2014).

[29] C. L. Kane and E. J. Mele, Phys. Rev. Lett. 95, 146802 (2005); 95, 226801 (2005).

[30] M. Z. Hasan and C. L. Kane, Rev. Mod. Phys. 82, 3045 (2010); X.-L. Qi and S.-C. Zhang, ibid. 83, 1057 (2011).
[31] S. M. Girvin and A. H. MacDonald, in Perspectives in Quantum Hall Effects, edited by S. Das Sarma and A. Pinczuk (Wiley, New York, 1997); D. H. Lee and C. L. Kane, Phys. Rev. Lett. 64, 1313 (1990); S. L. Sondhi, A. Karlhede, S. A. Kivelson, and E. H. Rezayi, Phys. Rev. B 47, 16419 (1993); K. Moon, H. Mori, K. Yang, S. M. Girvin, A. H. MacDonald, L. Zheng, D. Yoshioka, and S.-C. Zhang, ibid. 51, 5138 (1995).

[32] H. A. Fertig, L. Brey, R. Côté, and A. H. MacDonald, Phys. Rev. B 50, 11018 (1994).

[33] V. Mazo, H. A. Fertig, and E. Shimshoni, Phys. Rev. B 86, 125404 (2012).

[34] V. P. Gusynin, V. A. Miransky, S. G. Sharapov, and I. A. Shovkovy, Phys. Rev. B 77, 205409 (2008).

[35] Kun Yang, S. Das Sarma, and A. H. MacDonald, Phys. Rev. B 74, 075423 (2006). 